\title{
Effect of Prestrain on the Fatigue Life of Superelastic Nitinol
}

\author{
Karthikeyan Senthilnathan, Ali Shamimi, Craig Bonsignore, Harshad Paranjape, and Tom Duerig
}

(Submitted August 14, 2018; in revised form June 15, 2019; published online September 23, 2019)

\begin{abstract}
Three types of fatigue testing are performed to elucidate the effects of prestraining superelastic Nitinol on its subsequent fatigue lifetime: rotary bending and tension-tension testing of wire, and beam bending using diamond-shaped specimens fabricated from tubing. Results show that local plastic deformation during prestraining induces residual stresses that have a pronounced effect on fatigue performance, enhancing performance when the fatigue duty cycle is of the same sense as the prestraining (tensile prestraining followed by a tensile duty cycle, for example), and decreasing fatigue lifetime when the sense of the duty cycle is opposite to that of prestraining. This provides an avenue to increasing fatigue lifetime, but more importantly it highlights the need to fully understand the nature of the duty cycle: for example, prestraining a stent by crimping it into a delivery catheter induces favorable residual stresses with respect to subsequent pulsatile fatigue, but might accelerate fracture in other modes, such as axial or crush fatigue. Caution is also advised when trying to apply data from "constant life diagrams" derived from the literature (Ref 1, 2 for example) that may not properly reflect the strain history of the device being analyzed.
\end{abstract}

Keywords fatigue, inclusions, Nitinol, prestrain, residual stress

\section{Introduction}

The cyclic deformation of a superelastic Ni-Ti (Nitinol) medical implant is often defined by a mean strain and a cyclic strain amplitude (collectively called the duty cycle). Before imposing a duty cycle upon a device, most devices are initially deformed to a strain greater than the maximum it will experience in service (the mean strain plus the strain amplitude). For example, a stent is often crimped into a catheter that is substantially smaller than the artery into which it is to be deployed. This one-time strain, as illustrated in Fig. 1, is often called the prestrain. The effects of this prestrain on fatigue life are often ignored when analyzing and testing devices.

Theoretically, prestrain can have both detrimental and beneficial effects on fatigue life. Surface roughening and microcracks compromise durability (Ref 3,4$)$, while improvements can be expected from a reduced cyclic modulus (Ref 5) and increased hysteresis (Ref 6), both extending the elastic scope of the duty cycle and avoiding damage associated with martensite interface movement. Prestrain can also create residual stresses, which can either increase or reduce fatigue life. These residual stresses can result from non-uniform deformations such as bending or torsion, but as will be shown in this work, they can also result from microstructural inhomogeneities such as inclusions and voids. And while not specifically addressed in this work, one should be aware of the

Karthikeyan Senthilnathan, Ali Shamimi, Craig Bonsignore, Harshad Paranjape, and Tom Duerig, Confluent Medical Technologies, 47533 Westinghouse Drive, Fremont, CA 94539. Contact e-mail: karthiksen117@gmail.com. fact that grain anisotropy may lead to residual stresses (Ref 7, 8).

Experimentally, researchers have reported that increasing prestrain results in a beneficial improvement in cyclic life of Nitinol (Ref 9, 10). A microstructure-based study showed that strains up to $8-9 \%$ develop microstructures that assist both forward and reverse transformation of Nitinol which lead to improvement in subsequent fatigue life (Ref 11). Tensile fatigue tests on a superelastic wire when trained to different prestraining stresses before subjecting to fatigue tests result in significant improvement in low-cycle fatigue, due to dislocation-induced residual stresses that subsequently aid the phase transformation by reducing the yield stresses (Ref 12). On the other hand, at least one study showed no beneficial effect or even a slight decrease ( Ref 13). The intent of this study is to further characterize the effect of prestrain on superelastic Nitinol fatigue and more specifically to examine how the influence of prestrain depends upon the sequence and mode of loading.

Results from three types of fatigue test will be reported in this paper:

Rotary bending fatigue ( $R B F$ ) will be presented first. For this testing sequence, prestrain will be imposed in tension, subsequently followed by fatigue testing at $0 \%$ mean strain. Because of its experimental simplicity, this technique has become the most common way to characterize fatigue, and to the authors' knowledge, all prior studies on prestrain effects have employed this method. But the interpretation of RBF results is limited because one has no control over the ratio of minimum strain to maximum strain. Moreover, only the outer skin of the samples is exposed to the maximum tensile strain, so RBF tends to provide an optimistic perspective of lifetime. Test geometries are also normally limited to wire specimens, which may not accurately represent Nitinol laser-cut medical implants.

Tension-tension fatigue cycling will be presented next. For this testing sequence, prestrain is imposed in tension, sub- 


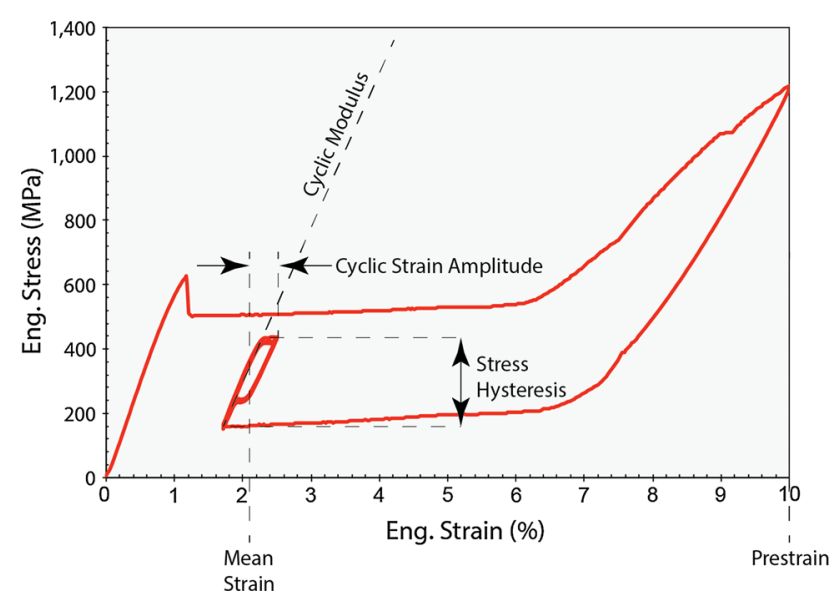

Fig. 1 A typical superelastic medical application consists of an initial prestrain ( $10 \%$ in this case) followed by unloading to the duty cycle characterized by a cyclic mean strain $(2.1 \%)$ and a cyclic strain amplitude $(0.40 \%)$

sequently followed by fatigue testing at $2 \%$ mean strain. This testing methodology delivers the most conservative description of fatigue lifetime since the entire sample gauge volume is uniformly exposed to tension. While experimentally difficult to run, the results are straightforward to interpret. Tension is a macroscopically uniform deformation so residual stresses are entirely due to microstructural inhomogeneities and anisotropy and thus uniformly distributed.

Diamond-shaped specimen flexion is the final form of fatigue we will consider, consisting of four beams exposed to bending. For this testing sequence, prestrain is imposed both in tension and in compression, followed by fatigue testing at $3.5 \%$ mean strain. Like rotary bending, the strains are highly localized and thus provide an optimistic perspective of lifetime. Both experimental methods and interpretation are complex requiring computational methods to calculate strains from global displacements. However, this geometry allows one to reverse the sense of the prestrain with respect to the duty cycle strain. This, in turn, allows us to introduce and compare residual stresses of the same sense and of opposite sense as those of the duty cycle, thus isolating the effects of residual stresses.

The experimental methodology and results of each of these three approaches will be individually discussed, followed by a discussion incorporating the results of all three test methods. Each fatigue test was done at a single, unique mean strain, and the effect of mean strain was not considered as part of this study.

\section{Rotary Bending Fatigue}

\subsection{Experimental}

Testing was conducted on a 50.8 atomic percent nickel straightened wire with a diameter of $0.28 \mathrm{~mm}$. The mechanical properties were adjusted through thermal aging to achieve a UTS of $1330 \mathrm{MPa}$, and upper and lower plateaus of 545 and $340 \mathrm{MPa}$ at $37^{\circ} \mathrm{C}$, respectively. Also, the transformation temperatures, measured by differential scanning calorimeter (DSC) per ASTM F2004 (Ref 14), were $M_{\mathrm{p}}=-80^{\circ} \mathrm{C}$, $M_{\mathrm{p}}^{*}=-9{ }^{\circ} \mathrm{C}$, and $A_{\mathrm{f}}=+8{ }^{\circ} \mathrm{C}$ (following the notation convention set forth in Ref 15 representing martensite formation peak, martensite reversion peak and austenite finish temperatures, respectively). The thermal aging process and transformation temperatures are representative of typical medical grade wires used for medical device applications. Prior to testing, wires were prestrained in tension to various strain levels at room temperature using an Instron equipped with a video extensometer. High-cycle fatigue tests were conducted to a maximum of $10^{6}(1 \mathrm{MM})$ cycles* at a frequency of $20 \mathrm{~Hz}$ in a temperature-controlled water bath at $37{ }^{\circ} \mathrm{C}$ with a minimum of three wires per strain level. Readers are referred to Ref 16 for detailed description of the test setup used for this study. Strain amplitudes were estimated $\left(\varepsilon_{\mathrm{a}}\right)$ based on the wire diameter $(d)$ and the radius of curvature of the mandrels $(\rho)$ through the expression $\varepsilon_{\mathrm{a}}=d / 2 \rho$.

\subsection{Results}

Figure 2 shows the strain amplitude vs. number of cycles fatigue (S-N curve) comparison for the specimens subjected to fully reversed bending without prestraining and after various tensile prestrains. The data demonstrate that fatigue behavior is strongly influenced by prestraining: with no prestrain, runouts are consistently observed at $0.6 \%$ strain amplitude, while the 8 and $10 \%$ prestrain conditions consistently ran out at 1.0 and $1.1 \%$ strain amplitude, respectively, corresponding to a 67 and $83 \%$ increase in tolerable strain amplitude. The effect of prestrain in improving fatigue life can be seen even at lower prestrains of 4 and $6 \%$, even though macroscopic plasticity is not observed. Such improvements are consistent with prior published results (Ref 9-12), but seemingly inconsistent with others (Ref 13). One must take care, however, in that the latter testing (Ref 13) was prestrained in bending, not tension. The importance of this will be discussed later.

\section{Tension-Tension Fatigue}

\subsection{Experimental}

The tests were performed on the same wire as mentioned in the previous section. The midsection of wires was reduced from $0.28 \mathrm{~mm}$ to $0.22 \mathrm{~mm}$ in diameter through electropolishing to create dog-bone specimens. Prior to testing, specimens were prestrained in tension to various strains $(4,6,9,10$ and $11 \%$ ) using an extensometer to confirm the prestrain level in the gauge section. Prestraining to $11 \%$ and beyond was challenging as the samples began to neck. Hence, a minimal number of samples were tested at the $11 \%$ prestrain condition. After prestraining, the specimens were released to a $2 \%$ tensile strain and cycled at various strain amplitudes holding the mean strain at $2 \%$. Testing was performed in water at $37{ }^{\circ} \mathrm{C}$ with a minimum of five samples at each strain amplitude. Cycling was conducted to $10^{7}(10 \mathrm{MM})$ cycles at $24 \mathrm{~Hz}$, at which time it was defined as a runout.

\footnotetext{
*For practical reasons, $1 \mathrm{MM}$ cycles were chosen as an endpoint for this series of tests. 1, 10, 100 and 400MM are commonly used high-cycle fatigue (HCF) test endpoints, and the authors caution against considering any of these endpoints a true "endurance limit".
} 


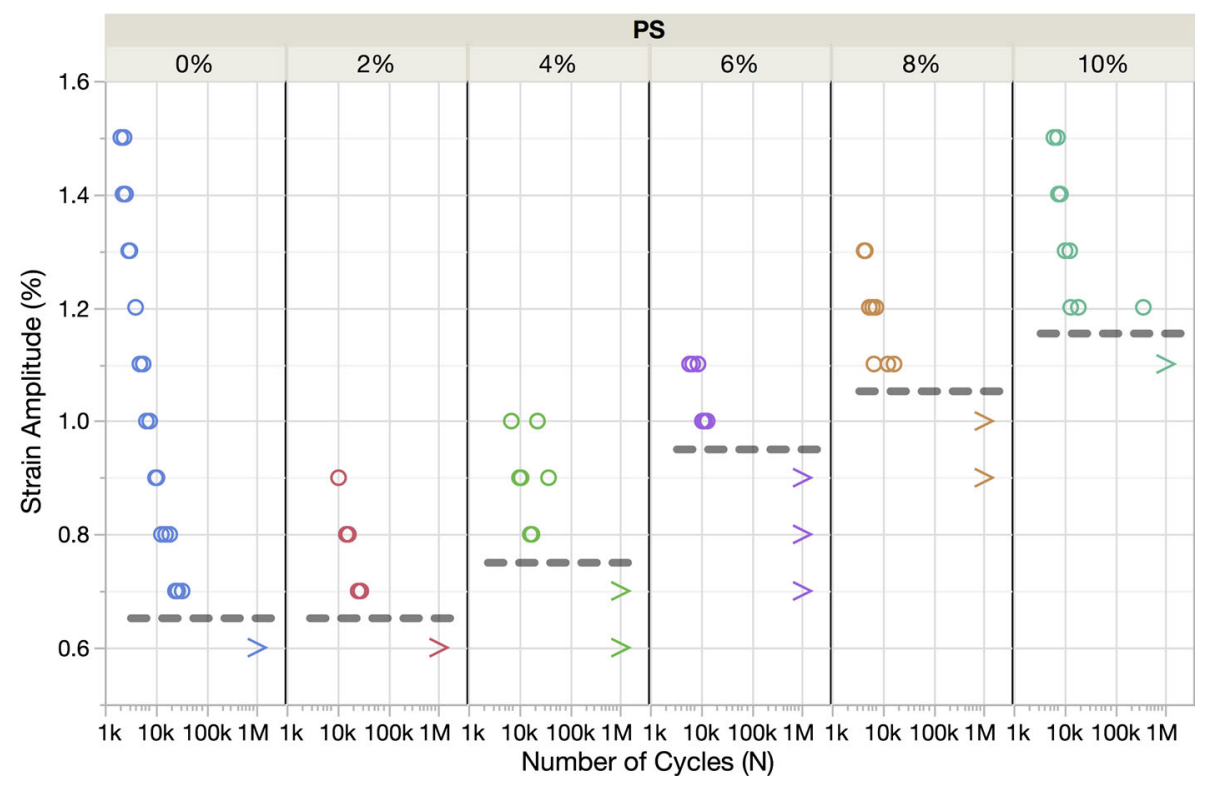

Fig. 2 Strain amplitude versus cycles to failure (S-N) curve for Nitinol wire specimens subjected to rotary bend fatigue under different prestrain conditions. Specimens were tested up to $10^{6}$ cycles. The circle symbols in the figure represent fractures, while the chevron symbols represent runouts. Dashed line separates fractures from runouts illustrating the trend of increasing durability with increasing prestrain (PS)

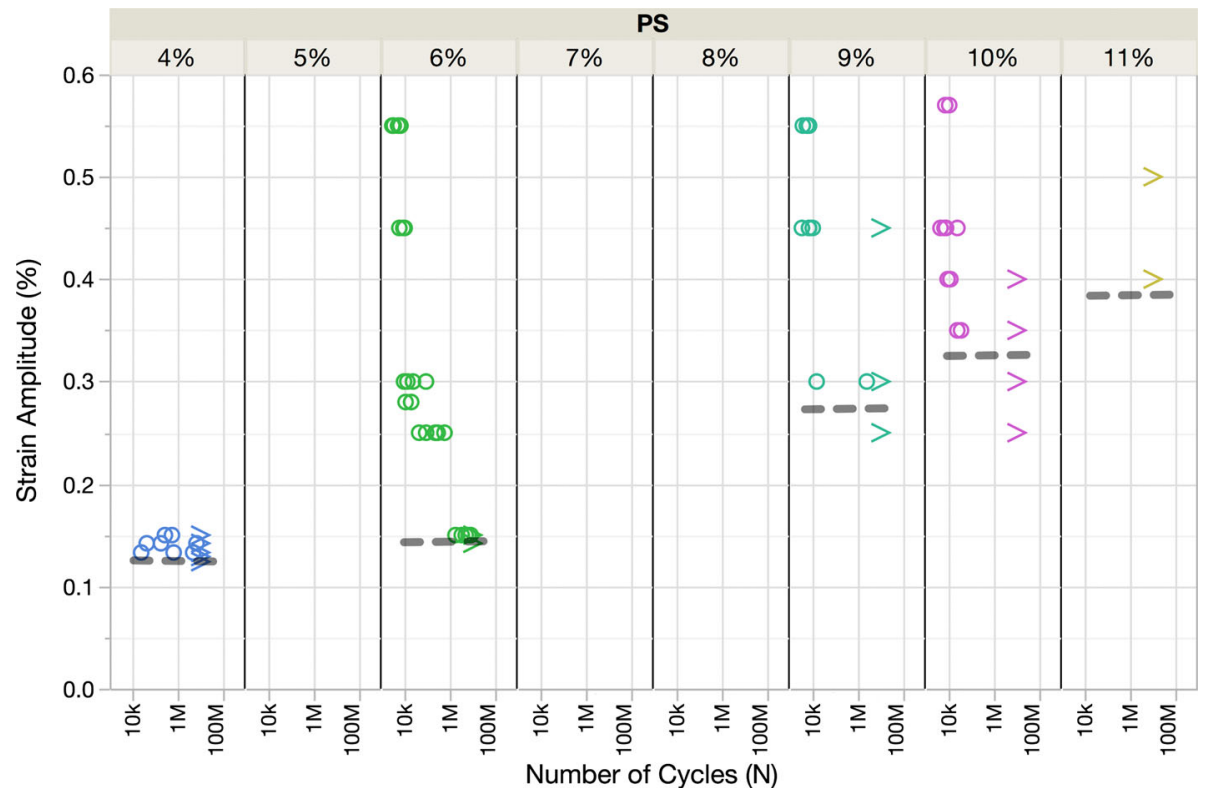

Fig. 3 Strain amplitude vs. number of cycles to failure (S-N) curve for Nitinol wire specimens subjected to tension-tension fatigue under different prestrain (PS) conditions. Specimens were tested to $10^{7}$ cycles. The circle symbols represent fractures, while the chevron symbols represent runouts. Dashed line separates fractures from runouts illustrating the trend of increasing durability with increasing prestrain. The prestrain columns at 5, 7 and $8 \%$ are intentionally left blank to help readers interpolate the results

\subsection{Results}

Tension-tension fatigue results (Fig. 3) show increased lifetimes with prestrain that are generally similar to that found in rotary bending. Runout strains are substantially lower in tension-tension loading compared with rotary bending because the former subjects the entire test specimen volume to the maximum strain amplitude rather than just the surface, increasing the probability of there being a critical defect. The magnitude of the improvement due to prestraining, however, is substantially greater than in rotating bending. Also, prestrain plays a prominent role in improving the low-cycle fatigue life of Nitinol when subjected to tensile fatigue (Ref 12) due to transformation-induced plasticity that produces dislocationinduced local residual stresses which lead to increase in fatigue lifetime.

In this tension-tension specimen, strain localization (socalled Lüders bands) expands to encompass the gauge volume as tensile strains increase to about $6 \%$. Increasing strain beyond $6 \%$, martensite elasticity is experienced up to about $8 \%$ strain, followed by plasticity with increasing strain. At strains 
exceeding $8 \%$, the effects of plasticity apply uniformly throughout the gauge volume, which may explain the durability benefit observed at these elevated prestrain levels.

\section{Diamond-Shaped Specimen Testing}

\subsection{General Background}

The diamond-shaped specimen used in this study is shown in Fig. 4. The intrados and extrados marked in that figure experience inverse stress states when the diamond is opened or closed. Figure 5, for example, shows the stress states that arise when the diamond is closed. (Details of the finite-element modeling are discussed in the subsequent section.) This action (closing the diamond) represents the most common scenario encountered in medical implants: a stent crimped into a catheter, for example. In the case of Fig. 5, the diamond is crimped until the maximum principal strain reaches $9 \%$ (a displacement of $4.7 \mathrm{~mm}$ ). At the end of crimping, the maximum tensile strain and stress are found in the region of the extrados, while the maximum compressive strains and stresses are observed at the intrados (see inset at lower right). Importantly, if the crimping is sufficient to cause plastic deformation at the extrados, then releasing the load on the diamond results in residual stresses of the opposite sense: continuing the example above, a stent that is deployed freely from a constraining catheter results in residual compressive stresses at the extrados and residual tensile stresses at the intrados (inset at lower left). Had the diamond instead been stretched open for prestraining, the stresses in Fig. 5 would be of a reverse sense: tension at the intrados during deformation leading to compressive residual stresses upon release.

Returning to the above example of a stent compressed into a delivery catheter, in practice the stent is not freely deployed, but rather deployed into a vessel that constrains it at some diameter smaller than its free diameter: from there, a duty cycle is imposed, defined by a mean strain state and a cyclic strain. Stents are constrained in a radially compressed state; thus, the sense of stresses and strains at the extrados is of the same sense as the initial prestrain. From a testing perspective, however, one can also apply a duty cycle of the opposite sense: prestraining by closing the diamond (creating a tensile stress state at the extrados), but then stretching the diamond to impose tension at the intrados. This scenario is shown in Fig. 6. This ability to apply a duty cycle of the same or opposite global displacement as the prestrain allows one to reverse the residual stress state which will help to determine whether residual stresses are dominant in modifying the fatigue lifetime of superelastic Nitinol.

There are thus four possible loading scenarios as outlined in Table 1. Two of the four create compressive residual stresses at the locations that will be exposed to tension during cycling (conditions $\mathrm{O}-\mathrm{O}$ and $\mathrm{C}-\mathrm{C}$ ), and two create tensile residual stresses at those locations $(\mathrm{C}-\mathrm{O}$ and $\mathrm{O}-\mathrm{C})$. If residual stresses are controlling fatigue life, we expect the former situation to extend fatigue life and the latter to accelerate fracture.

\subsection{Experimental}

The material used in this study was ultra-high-purity $\mathrm{Ni}_{50.8} \mathrm{Ti}_{49.2}$, also known as ELI (extra low interstitial), with a total $\mathrm{C}+\mathrm{O}+\mathrm{N}$ content of below $100 \mathrm{wppm}$. The diamond specimens shown in Fig. 4 were laser-cut from an $8.0 \mathrm{~mm}$ OD $\times 7.0 \mathrm{~mm}$ ID tube and expanded in multiple deformation and thermal aging steps to achieve a UTS of $1210 \mathrm{MPa}$, and upper and lower plateaus of 404 and $134 \mathrm{MPa}$ at $37{ }^{\circ} \mathrm{C}$, respectively. Also, the transformation temperatures measured by DSC per ASTM F2004 were $M_{\mathrm{p}}=-53^{\circ} \mathrm{C}, M_{\mathrm{p}}^{*}=+12^{\circ} \mathrm{C}$, and $A_{\mathrm{f}}=+20{ }^{\circ} \mathrm{C}$. The thermal aging process and transformation temperatures are representative of typical laser-cut medical devices. After electropolishing, the samples were measured and dimensionally characterized to assure accurate determinations of strain from displacements using finite-element analysis (FEA).

The finite-element model of the diamond specimens was meshed with C3D8R elements. The geometry was discretized with a mesh density of $12 \times 12$ elements across the strut width and wall thickness to determine the resulting stresses and strains. The $12 \times 12$ mesh density is representative of the mesh discretization used in modeling (including submodels) of typical medical devices that provides optimal solution. The final ABAQUS User Material (UMAT) properties of the aged

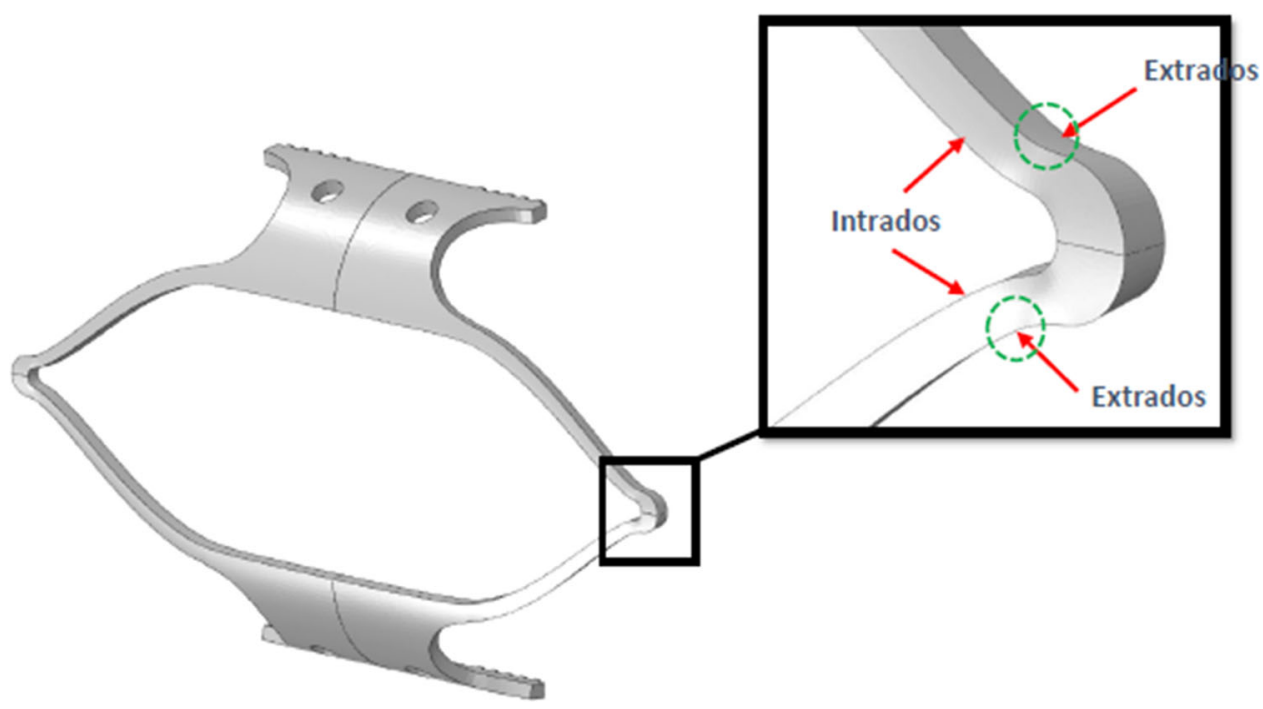

Fig. 4 Shape set diamond-shaped specimen geometry with the inset showing the intrados and extrados region of the struts 


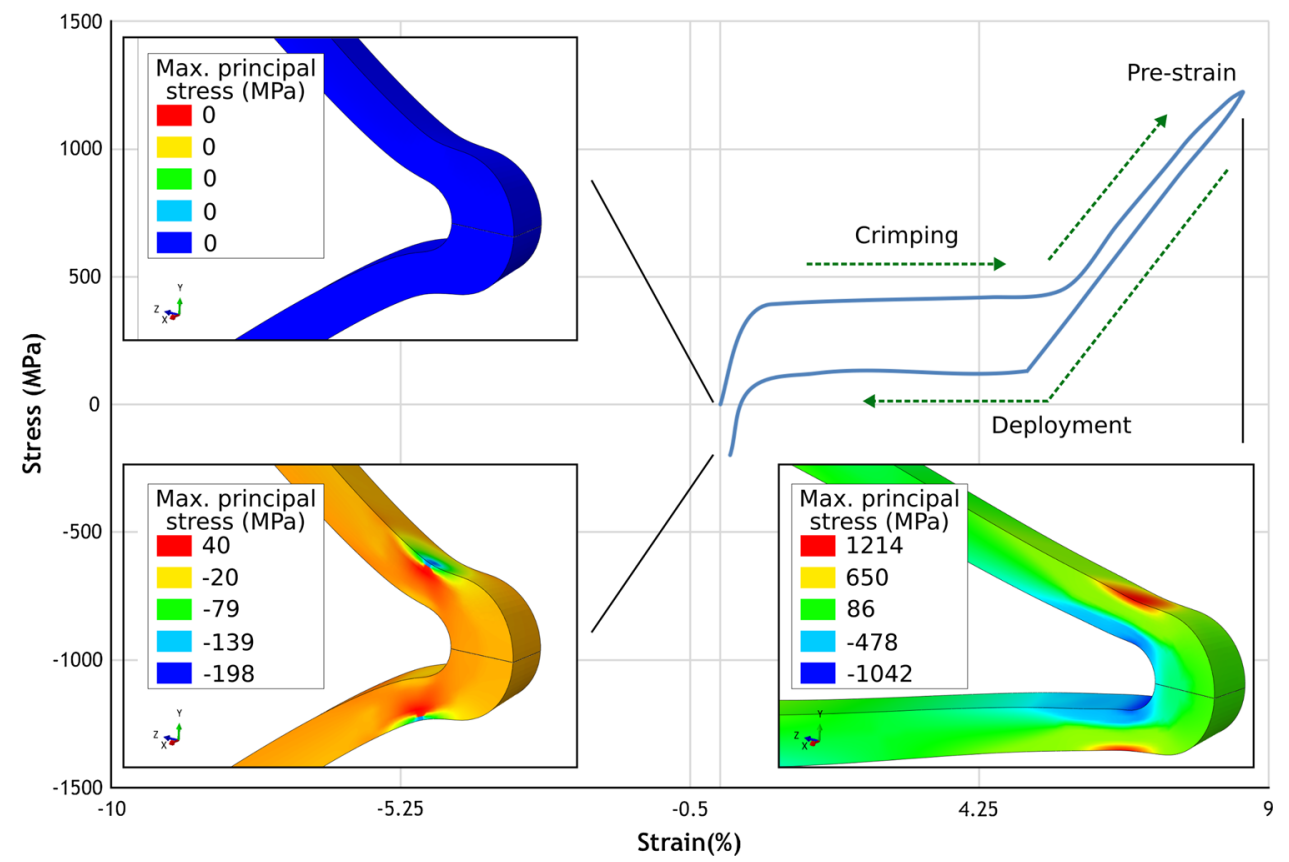

Fig. 5 Prestraining by closing the diamond results in tensile stresses at the extrados and compressive stresses at the intrados (lower right). Releasing the load from the diamond results in compressive residual stress at the extrados and tensile residual stress at the intrados (lower left). The stress-strain curve in the background represents the stresses and strains experienced by the element that ultimately reaches the largest strain value

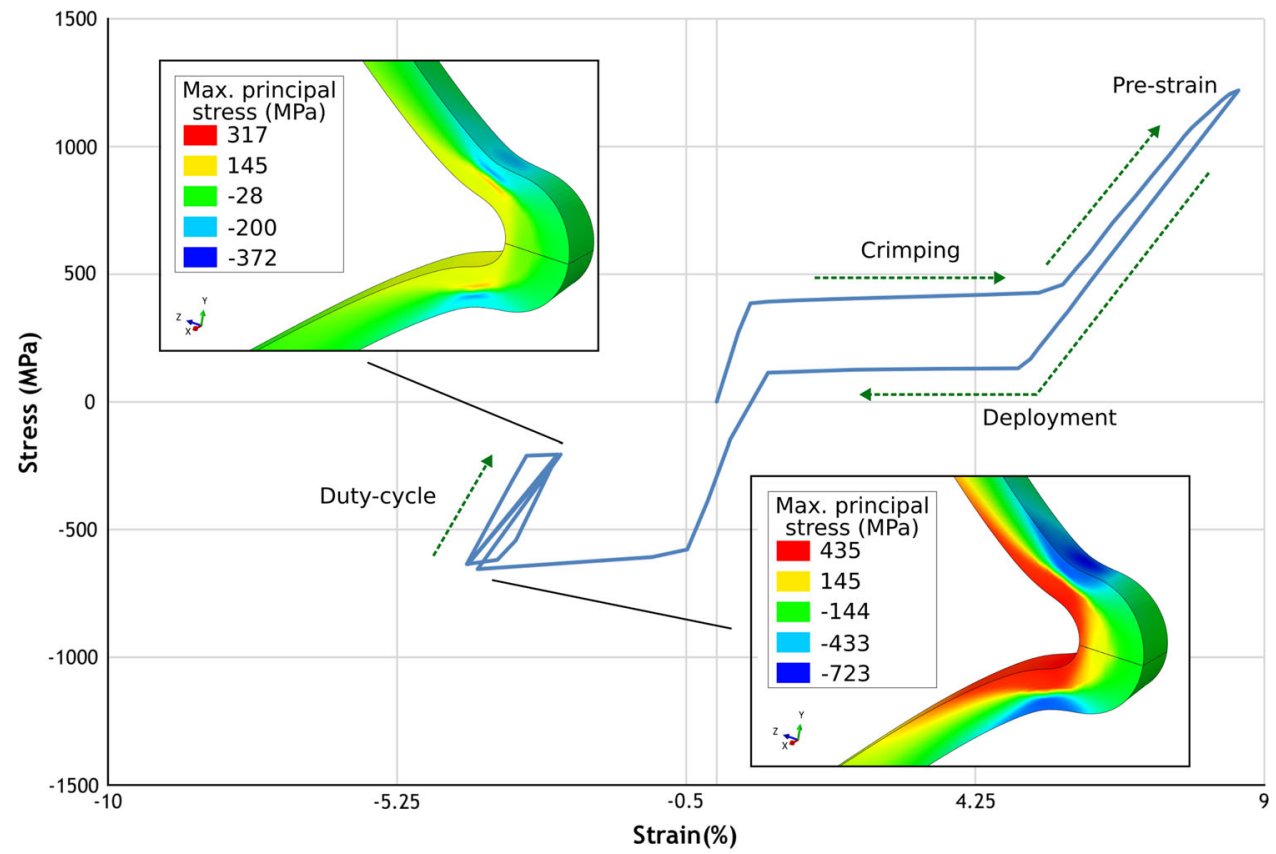

Fig. 6 After the tensile prestrain at the extrados shown in Fig. 5, the diamond is unloaded and then stretched. A cyclic duty cycle is then imposed with mean strains opposite in sense (compressive) to those experienced during prestraining

material were determined from tensile testing of coupon samples subjected to heat treating representative of the diamond specimens. The key ABAQUS UMAT parameters are shown in Table 2, and for tension-compression asymmetry, a ratio of 1.5 (start of transformation stress in compression/start of transformation stress in tension) was assumed representative of typical Nitinol tubes (Ref 17). Sensitivity to material parameters was not analyzed as part of this study, as the primary intent is to understand the interaction of prestresses on fatigue life and not on the absolute value of the stresses/strains.

Testing was performed on a 12-station Instron E-3000 machine with load cells on each station. Six diamond samples were tested for each of the four combinations. Since fixation is rigid (Fig. 7), a fracture on one side of the diamond does not influence the conditions experienced by the other. Thus, each 
Table 1 Four possible permutations of prestrained testing for diamond-shaped specimens

\begin{tabular}{lccccc}
\hline & \multicolumn{2}{c}{ Prestrain } & & Release & Duty cycle \\
\cline { 2 - 3 } $\begin{array}{l}\text { Test } \\
\text { condition }\end{array}$ & $\begin{array}{c}\text { Diamond } \\
\text { displacement }\end{array}$ & $\begin{array}{c}\text { Tensile stress } \\
\text { location }\end{array}$ & & $\begin{array}{c}\text { Residual compression } \\
\text { location }\end{array}$ & $\begin{array}{c}\text { Tensile stress } \\
\text { location }\end{array}$ \\
\hline C-O & Close & Extrados & Extrados & Open \\
O-O & Open & Intrados & Intrados & Open \\
C-C & Close & Extrados & Extrados & Close \\
O-C & Open & Intrados & Intrados & Extradose \\
\hline
\end{tabular}

Table 2 ABAQUS UMAT parameters used to model the diamond specimens

\begin{tabular}{ll}
\hline ABAQUS parameters & Values \\
\hline Austenite elasticity, MPa & 76,000 \\
Martensite elasticity, MPa & 30,714 \\
Start transformation loading, MPa & 380 \\
End transformation loading, MPa & 430 \\
Start transformation unloading, MPa & 130 \\
End transformation unloading, MPa & 100 \\
Start transform stress compression, MPa & 570 \\
Ultimate tensile stress in yield curve, MPa & 1420 \\
Ultimate tensile strain in yield curve, \% & 14.0 \\
\hline
\end{tabular}

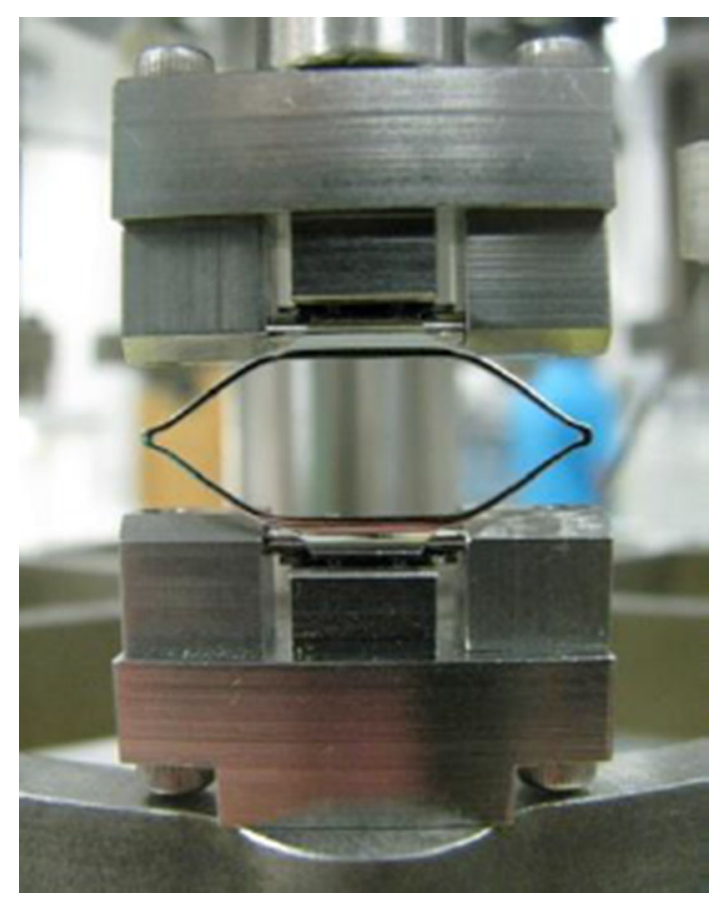

Fig. 7 Representative diamond specimen manufactured and mounted on custom-fabricated test fixtures

diamond can be considered to be two "V"-shaped test specimens. Hence, a total of 12 "V" specimens were tested per condition.

All samples were prestrained in a water bath controlled at $37{ }^{\circ} \mathrm{C}$ to a displacement corresponding to a $9 \%$ maximum strain (samples $\mathrm{C}-\mathrm{O}$ and $\mathrm{C}-\mathrm{C}$ by closing the diamond and samples $\mathrm{O}-\mathrm{O}$ and $\mathrm{O}-\mathrm{C}$ by opening). "Baseline" samples that were not prestrained were also included in the study. FEA was used to determine the required deformation and strains based on the actual part geometry. The fatigue strains (mean strain and strain amplitude) were computed using the tensor method by computing the average and differences of the principal values of the strain tensors at the extremes of the loading. It has been shown that both normal tensor method and modified tensor method consistently predict fracture or survival under different loading conditions for stents (Ref 18). The actual loaddisplacement curves for the four prestrain scenarios are shown in Fig. 8 with negative displacements corresponding to closing of diamonds and vice versa. Following the prestrain, all samples (including the reference samples) were stretched or closed to impart a $3.5 \%$ maximum mean strain and then cycled at $24 \mathrm{~Hz}$ with a strain amplitude of $0.75 \%$. If no fracture was observed after $10^{6}(1 \mathrm{MM})$ cycles, the test was considered a "runout" and the cyclic strain amplitude was increased while maintaining the same mean strain level. This was continued until a substantial number of fractures were observed. The baseline condition was only tested with a closing duty cycle (tension at the extrados), the most common scenario experienced by Nitinol medical devices. The experimental loaddisplacement of condition $\mathrm{C}-\mathrm{O}$ in Fig. 8 corresponds to the stress strain history for the diamond specimen shown in Fig. 6.

\subsection{Results}

As one would expect, scanning electron microscopy images (Fig. 9) verified that fractures initiated at the areas of highest tensile duty stress: the intrados of conditions $\mathrm{C}-\mathrm{O}$ and $\mathrm{O}-\mathrm{O}$ and the extrados of conditions $\mathrm{C}-\mathrm{C}, \mathrm{O}-\mathrm{C}$ and the reference sample. Table 3 summarizes the results of the testing with number of "V" specimen fractures specified - the numbers listed indicate the cumulative number of fractures from the total of twelve "V" specimens. As with previous testing, prestraining has a significant effect on lifetime, but here we see that the effect can be an enhancement or a degradation depending on the sense of the prestrain with respect to the duty cycle: conditions $\mathrm{O}-\mathrm{O}$ and $\mathrm{C}-\mathrm{C}$ performed better than the reference samples, while conditions $\mathrm{C}-\mathrm{O}$ and $\mathrm{O}-\mathrm{C}$ performed worse. This is consistent with the expectation that compressive residual stresses are protective when superimposed onto areas of tensile stress, and tensile residual stresses accelerate the fatigue process, regardless of whether the intrados or extrados is critical (tension bearing). These same results are plotted based on probability of survival in Fig. 10, with the baseline condition denoted by dashed green line. 


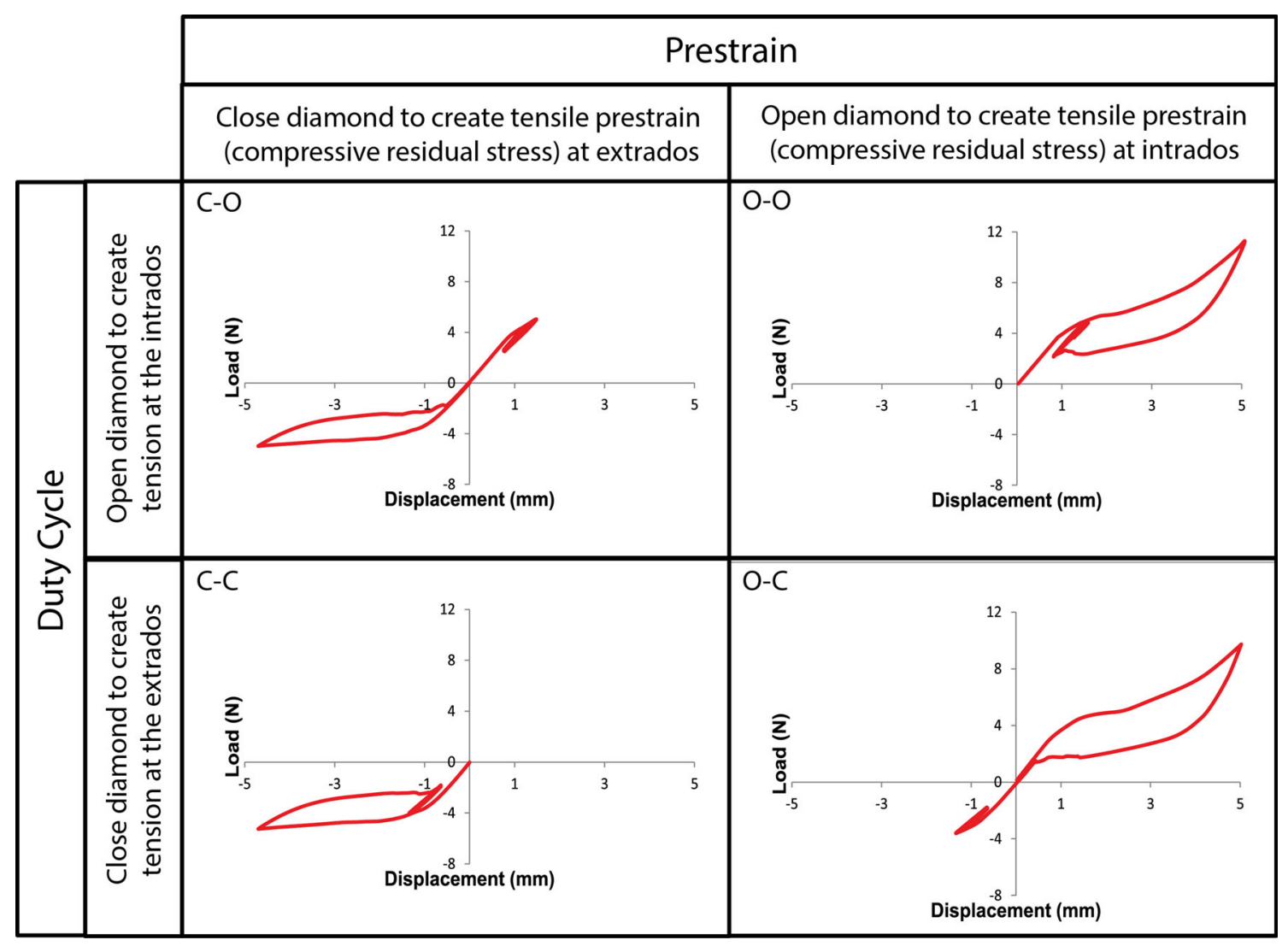

Fig. 8 Load-displacement curves for the four test conditions: (C-O) Load-displacement to achieve tensile prestress on extrados and tensile duty cycle stress on intrados. (O-O) Load-displacement to achieve tensile prestress on intrados and tensile duty cycle stress on intrados. (C-C) Load-displacement to achieve tensile prestress on extrados and tensile duty cycle stress on extrados. (O-C) Load-displacement to achieve tensile prestress on intrados and tensile duty cycle stress on extrados

Lifetimes in this test are substantially longer than those resulting from the previous two testing modes. This can be attributed to the fact that this study employed ultrapure material which is known to have a significantly longer lifetime (Ref 19), because the peak strains and strain amplitudes are experienced by a very small volume of material.

\section{Discussion}

The results of the diamond specimen testing demonstrate that non-uniform deformations such as bending or torsion create zones of residual compressive stress that have a pronounced effect on fatigue life. When the residual stresses are subtractive to a tensile duty cycle stress state, they protect, and when they are additive, they accelerate failure. In both the tension-tension and rotating bending experiments, however, the wires were prestrained in uniaxial tension, which, in an isotropic and homogeneous material, should produce no residual stresses. Yet there is still a pronounced influence. Of course, no material is isotropic and homogeneous, so microscopic residual stresses are expected to arise due to defects and grain orientation (Ref 7,8$)$. In the case of Nitinol, it is well established that in nonmetallic inclusions, both $\mathrm{TiC}$ and $\mathrm{Ti}_{2} \mathrm{NiO}_{\mathrm{x}}$ play a critical role in fatigue initiation (Ref 19-22). In fact, virtually all fatigue failures nucleate at easily identified carbide or oxide defects (Ref 20). It has also been shown that reducing the number and/or size of these defects has a pronounced effect on fatigue lifetime (Ref 19, 22).

FEA was used to model the interaction of prestrain, residual stresses, and the nucleation potency of nonmetallic inclusions. An FEA nonlinear code (ABAQUS/Standard version 6.13-2) with a user-defined material subroutine (UMAT) typical of the superelastic-plastic response of Nitinol (Table 4) was used to model a 0.22-mm-diameter wire with a 4-micron cubic inclusion representing a surface carbide as shown in Fig. 11. Surface carbides represent the vast majority of observed initiation sites, though surface oxides also play a role (Ref 23). The geometry was discretized with a very-fine 1-micron cubic mesh (C3D8R elements) both on the inside and around the inclusions to capture the effect of stress localization at the boundaries. $* *^{\dagger}$ The carbide was modeled as a linear isotropic material with Young's modulus of $210 \mathrm{GPa}$ and a Poison's ratio of 0.3 . Inclusions often separate from the surrounding matrix during hot working, leaving voids between the inclusion and the matrix. Accordingly, a void of the same geometry as the

\footnotetext{
**In this simulation, particles were tied to the surrounding matrix. Therefore, when the bulk material is stretched in tension, the particle is stretched as well, rather than separating from the bulk to create a void, as might occur in reality.

${ }^{\dagger}$ Due to the stress singularity at the boundary where inclusion is tied to the bulk material, mesh convergence is not possible, and hence, the geometry was discretized with a very-fine $1-\mu \mathrm{m}$ mesh size. The main intent of the simulation is to understand the interaction of inclusions with bulk material and not on the absolute values of stresses/strains.
} 

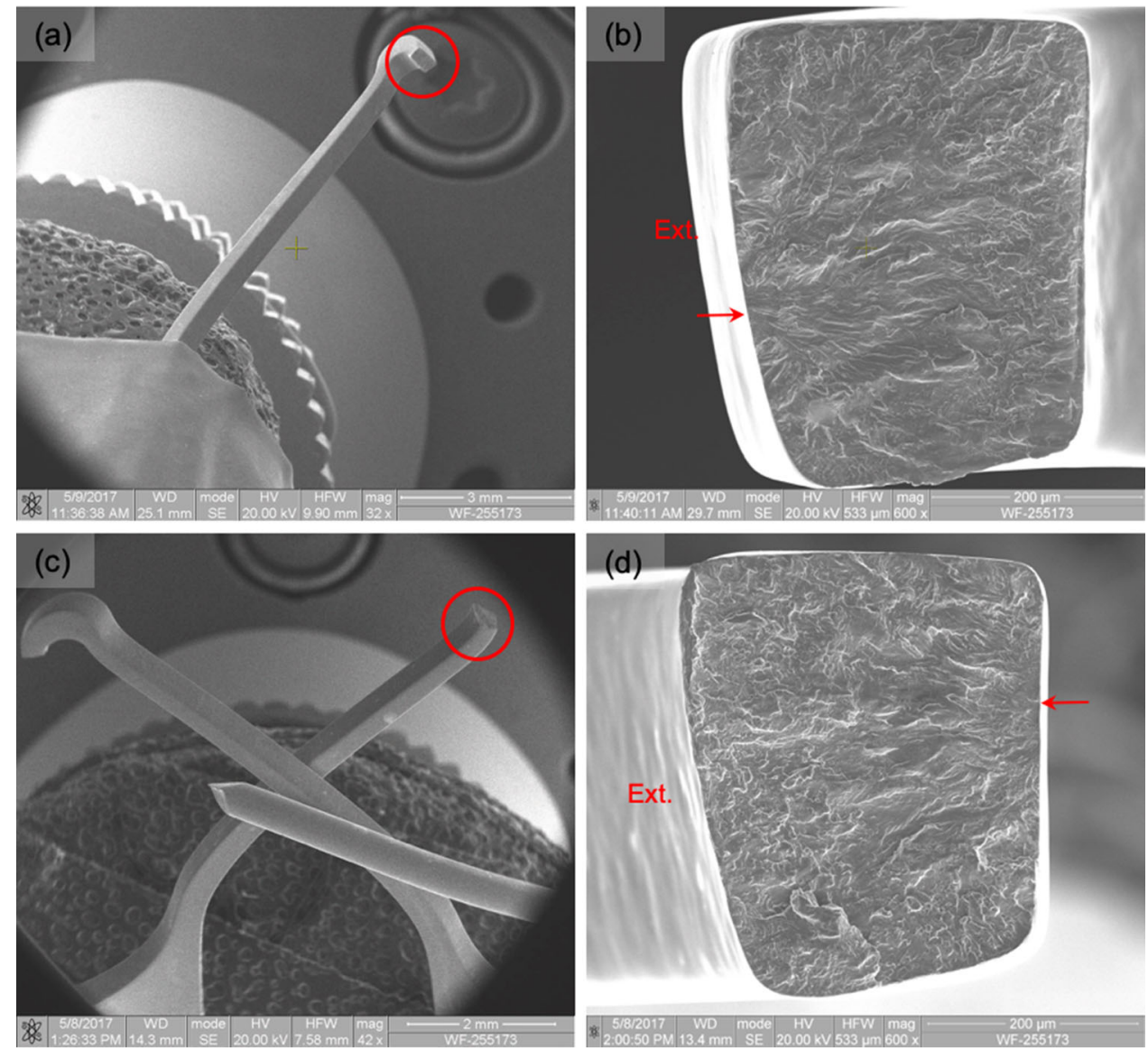

Fig. 9 Representative scanning electron microscopy images showing the fracture initiation locations from intrados and extrados (marked by arrows). (a) and (b), fracture initiated from the extrados (C-C condition); (c) and (d), fracture initiated from the intrados (O-O condition)

Table 3 Cumulative fractures from diamond fatigue testing out of 12 "V" specimens

\begin{tabular}{lccccc}
\hline Strain amplitude, $\%$ & Baseline & Condition C-O & Condition O-O & Condition C-C & Condition O-C \\
\hline 0.75 & 0 & 0 & 0 & 0 & 0 \\
1.30 & 0 & 3 & 0 & 0 & 1 \\
1.88 & 0 & 7 & 0 & 0 & \\
2.24 & 2 & 8 & 0 & 2 & \\
2.76 & 8 & $*$ & 2 & 3 & $*$ \\
2.90 & 11 & $*$ & 10 & 6 & $*$ \\
3.03 & $*$ & $*$ & $*$ & 8 & $*$
\end{tabular}

Asterisks $(*)$ represent testing was not performed since considerable number of specimens already fractured

inclusion was also modeled, providing two extreme cases. It has been reported that particle void assemblies (PVAs) are in fact the worst-case condition (Ref 22, 24) and warrant modeling in the future work. FEA studies have also shown that the morphology of the inclusions (size and shape) plays a role in influencing the fatigue life of Nitinol compared to inclusion material type and inclusion-inclusion interactions (Ref 24, 25).

The results of both a void and a carbide inclusion are presented in Fig. 12. Figure 12(a) represents the starting state for the void and inclusion scenarios with stresses set to same scale for comparison. For better visualization, the inclusion is hidden in the images on the right side and a half-symmetry cutout is shown to visualize the subsurface. When the wire is axially stretched to a $10 \%$ global strain, the maximum principal stresses occur around the inclusion or void as shown in Fig. 12(b). The location of maximum stress shifts between the two scenarios as indicated by the blue arrow. For the void, it occurs along the circumferential direction at the smallest cross section, and for the attached inclusion it occurs along the longitudinal direction. Upon release of the applied loading, the wire reverts to the starting state, and compressive residual stresses build up at the locations that experienced the maximum 


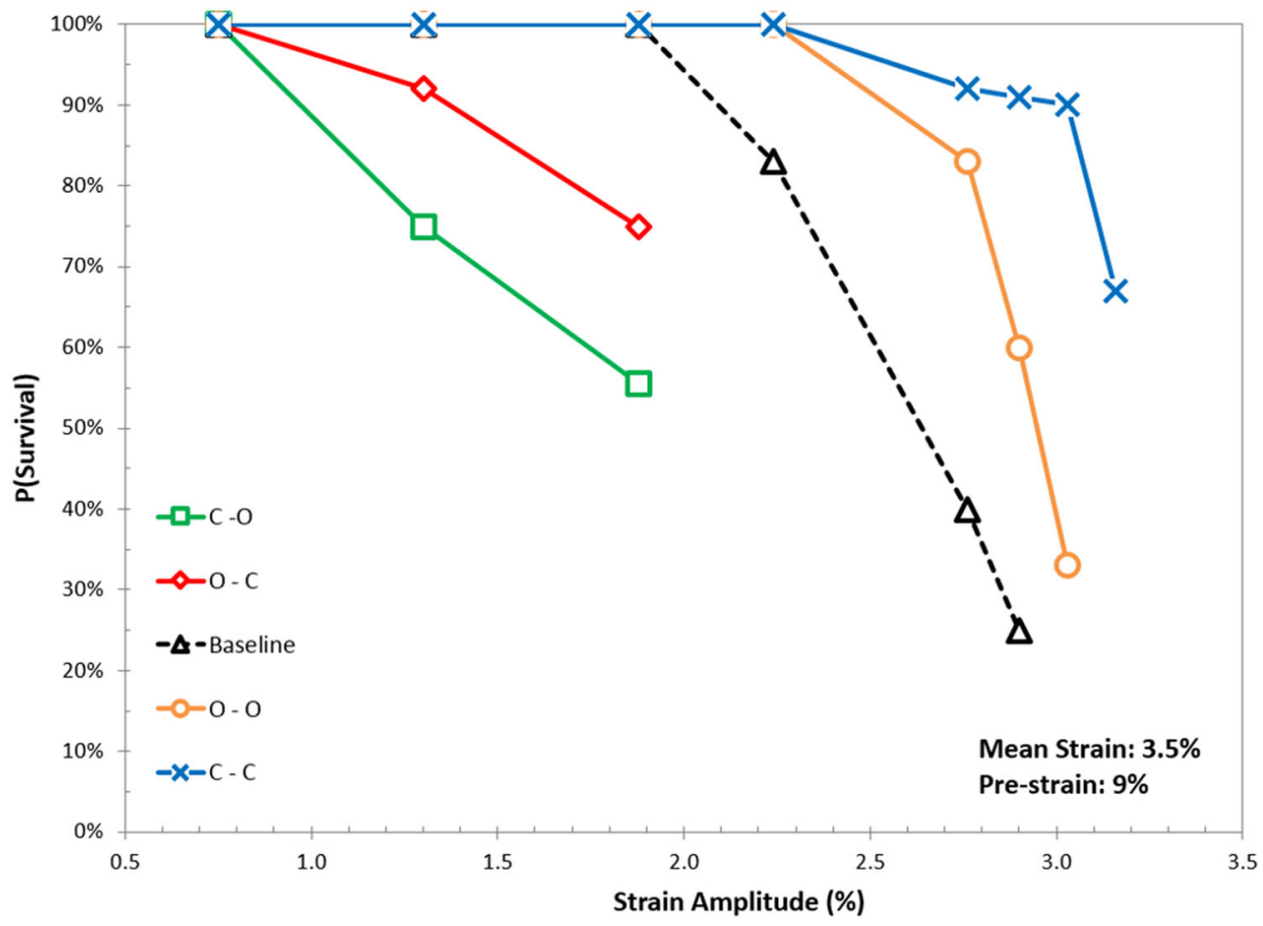

Fig. 10 Probability of survival plot based on strain amplitude for different conditions. The baseline condition is represented by dashed green line. The curves to the left indicate a decrease in fatigue life and to the right indicate an increase in fatigue life (Color figure online)

Table 4 ABAQUS UMAT parameters used for the wire in the embedded inclusion models

\begin{tabular}{ll}
\hline ABAQUS parameters & Values \\
\hline Austenite elasticity, MPa & 62,857 \\
Martensite elasticity, MPa & 27,778 \\
Transformation strain & 0.046 \\
Start transformation loading, MPa & 460 \\
End transformation loading, MPa & 500 \\
Start transformation unloading, MPa & 240 \\
End transformation unloading, MPa & 210 \\
Start transform stress compression, MPa & 690 \\
Ultimate tensile stress in yield curve, MPa & 1510 \\
Ultimate tensile strain in yield curve & 0.128 \\
\hline
\end{tabular}

tensile stress during loading as shown in Fig. 12(c). The magnitude of the compressive residual stresses is comparable for the two conditions, though the locations are distinctly different. The size and shape of the inclusions or void is expected to play a critical role in the magnitude of compressive residual stresses and the fracture initiation and crack growth direction. Authors are working on a separate study to highlight the importance of inclusion morphology and its effect on fatigue life.

Figure 13 highlights the protective nature of the residual stresses for both cases. In Fig. 13(a), a simple stretch to $6 \%$ strain is shown without a prior prestrain, while in Fig. 13(b), the same $6 \%$ stretch is shown, but here preceded by the $10 \%$ prestrain described in Fig. 12. While the maximum stresses for the void are greater in both cases, the maximum stress drops by $30 \%$ for voids and by $22 \%$ for inclusions after prestraining.
New, higher-purity alloys with fewer and smaller inclusions have recently become available, and as discussed above, these alloys have a distinctly longer fatigue lifetime. In light of these results, however, one would expect a greater performance difference between standard and ultrapure materials when prestrain is a minimum. On the contrary, if the prestrain is substantial, it should be expected to lessen the difference between the alloys, since prestrain casts a protective effect on inclusions. But even if one could produce a complete void-free and inclusion-free material, one should still expect an effect from prestrain: unfavorably aligned grains with a lesser transformational strain and higher plateau stress plastically deform on lower global strains than more ideally aligned neighbors (Ref 26). Just as with inclusions, these grains would be shielded by the ensuing residual stresses.

The benefits of residual compressive stresses to enhance the fatigue resistance of traditional metallic components have long been recognized (Ref 27-29). For example, many engineering components have been shot-peened or cold-worked with fatigue strength enhancement as the primary objective. The question now is whether the superelastic properties of Nitinol provide greater protection due to the large elastic energy that can be stored during a deformation. To gain some insight into this matter, the same wire and inclusion geometry was used, but the Nitinol UMAT was replaced by that of a conventional elastic material, namely Stainless Steel 316L. Here a generic material response for steel with cyclic plasticity was assumed for the material behavior. Figure 14(a) and (b) compares the residual stresses between steel and Nitinol with an attached inclusion and a void, respectively, after unloading from a $10 \%$ global tensile strain. The compressive residual stress values are significantly higher for Nitinol due to the stress-strain hysteresis, anticipating a greater improvement in fatigue performance. 


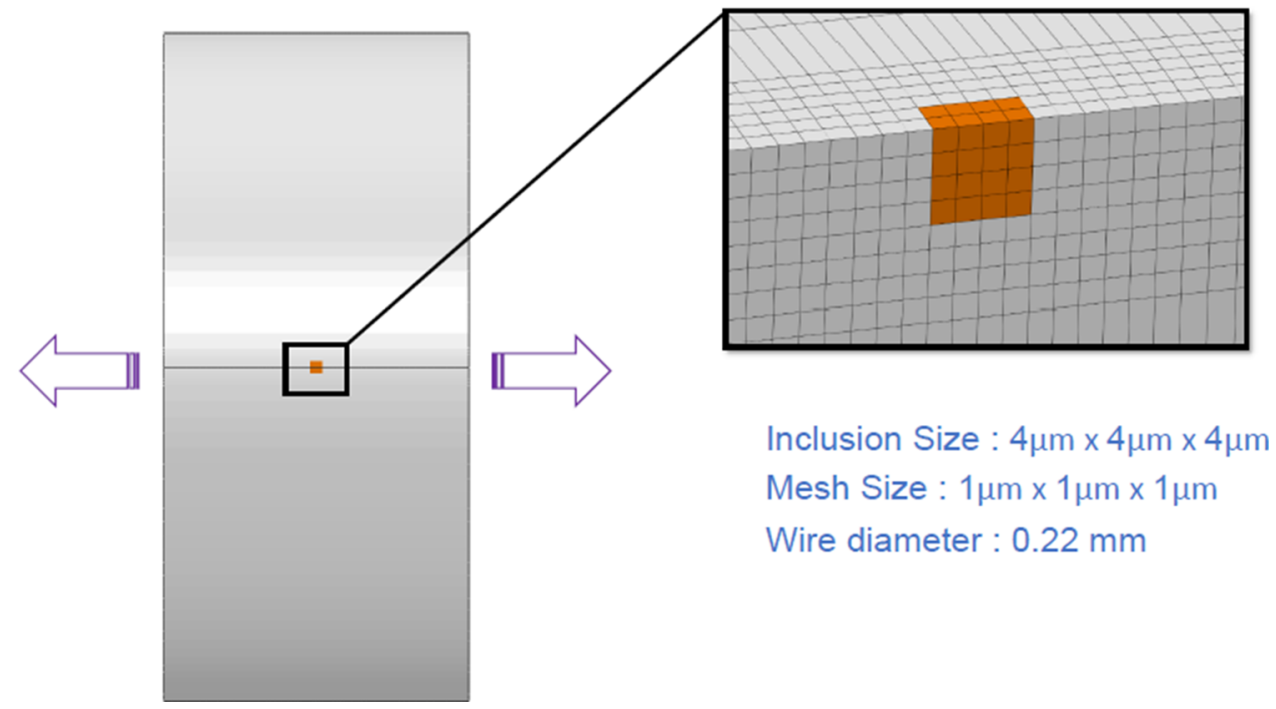

Fig. 11 Computational model of the wire specimen geometry (gray) with a surface inclusion (orange) and arrows indicating the loading direction. Inset shows the mesh discretization for the wire specimen and the inclusion (Color figure online)
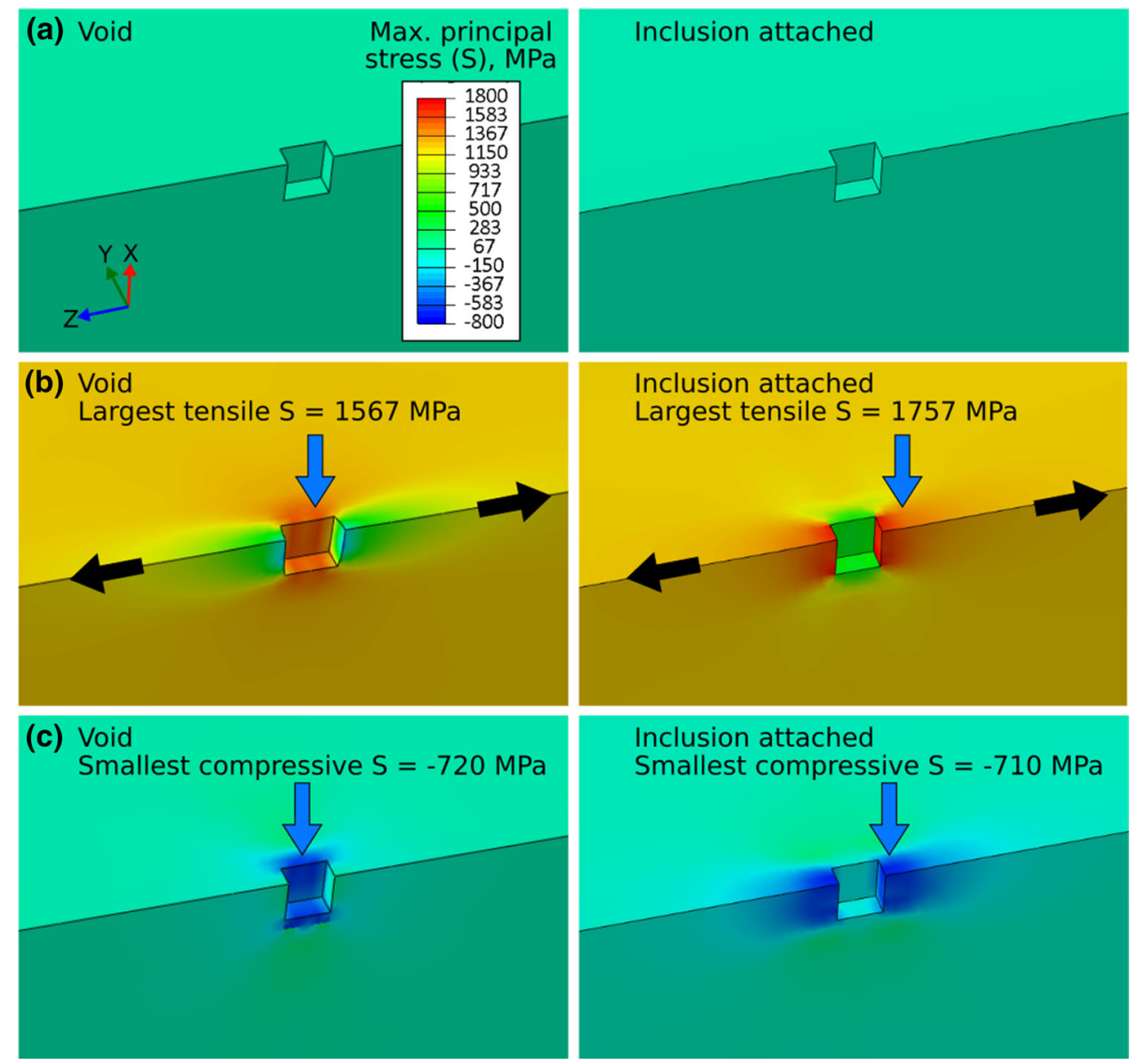

Fig. 12 Comparison of stress states for void vs. attached inclusion (inclusion removed to aid visualization). (a) Starting state of the geometry (left-represents void; right - represents inclusion). (b) Blue arrow representing the maximum principal stress locations at the end of loading to $10 \%$ global strain with the black arrows representing the loading direction (left-represents void; right-represents inclusion). (c) Residual stresses upon release of the applied loading (left—represents void; right—represents inclusion) (Color figure online)

We now turn our attention to the one previous report that showed no enhancement in rotating bending fatigue performance after prestraining in bending (Ref 13). In this case, prestraining was performed in bending, which creates both tensile and compressive residual stresses on the concave and convex sides, respectively. The applied duty cycle exposes all 

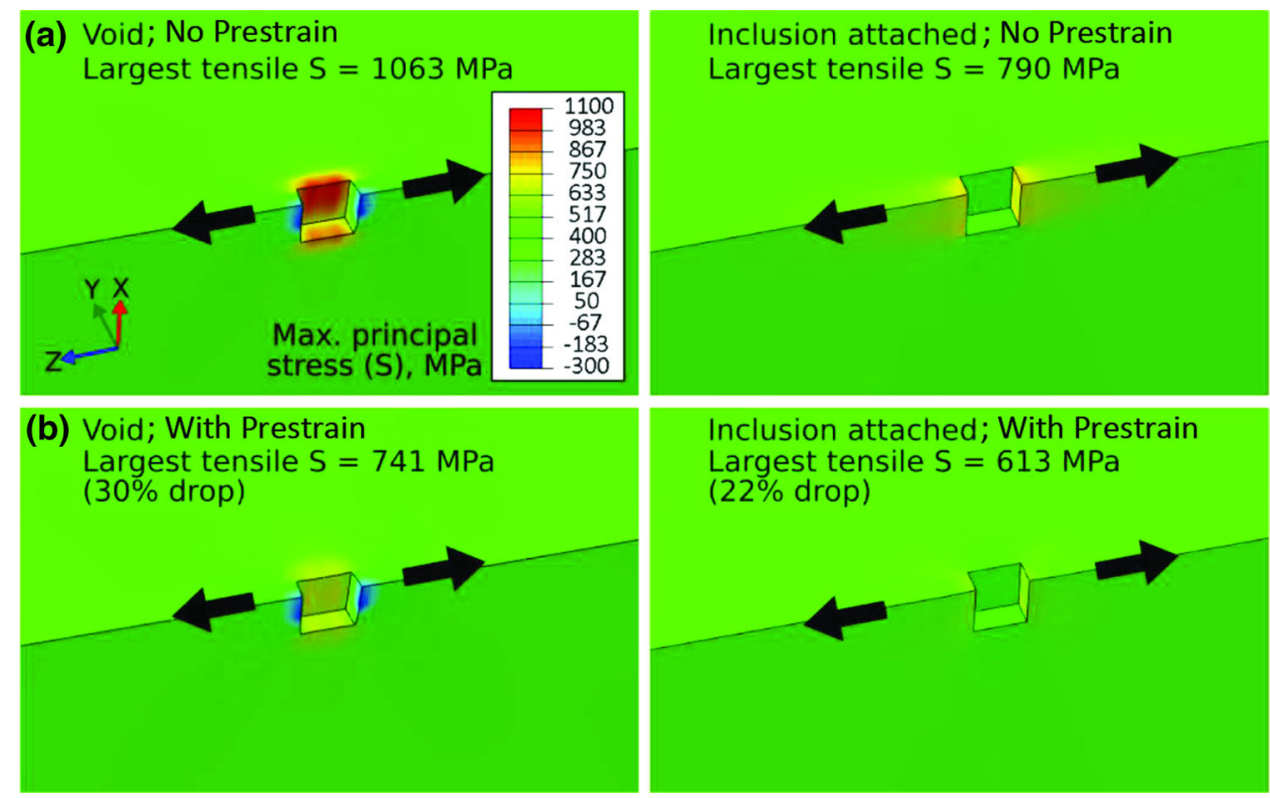

Fig. 13 Comparison of stress state for void vs. an attached inclusion (inclusion removed to aid visualization). (a) Maximum principal stress at the end of first pull to $6 \%$ indicating no prestrain (left—represents void; right—represents inclusion). (b) Maximum principal stress at the end of second pull to $6 \%$ after prestraining (left—represents void; right—represents inclusion)
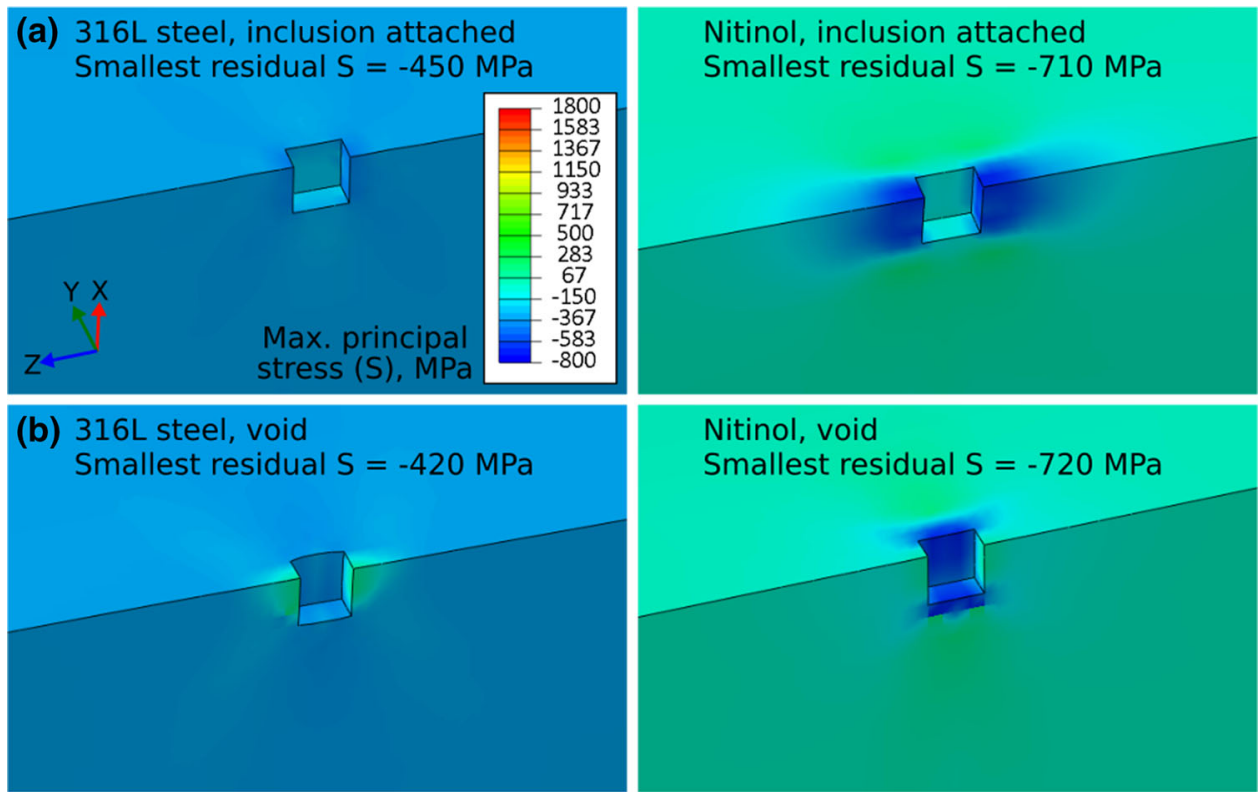

Fig. 14 Comparison of residual stress states for steel vs. Nitinol. (a) Maximum principal stress at the end of unloading for an attached inclusion (inclusion removed to aid visualization; left-represents steel; right—represents Nitinol). (b) Maximum principal stress at the end of unloading for a void (left—represents steel; right—represents Nitinol)

the surfaces to tension, including the surface in which tensile residual stresses have been installed. Thus, we should expect a deterioration in lifetime, exactly as reported.

In summary, whether prestrain introduces residual stresses due to non-uniform deformations, or defects, or less-ideally aligned grains, these residual stresses can protect or aggravate fatigue, and the magnitude of these residual stresses is enhanced by the elastic energy storage capacity of superelastic Nitinol. This has a few critical engineering implications. Firstly, fatigue testing can be highly deceptive: for testing to be predictive, samples must be appropriately prestrained, and generic S-N curves or constant life diagram from the literature are of little value. Secondly, this emphasizes how important it is to consider all deformation modes. For example, it was already shown that a stent crimped into a small catheter is protective to subsequent pulsatile duty cycles that produce tension at the stent extradoses. But, under different loading modes such as the axial fatigue the intradoses would be exposed to tension, where there is extant tensile residual stresses accelerating failure. Thus, depending on the deformation mode one type of fatigue 
is improved, while the other accelerated. Finally, one can easily envision that the highest cyclic strain areas in a device are protected by residual stresses, while an area with lower cyclic strains is not, making the lower cyclic strain location possibly the most fatigue critical and first to fracture. This has significant implications to safety factor analyses or when determining the location and thus the consequence of potential failures (Ref 30 ).

\section{Conclusions}

The effect of prestrain on fatigue life of Nitinol was analyzed in detail for different loading modes of rotary bend, tension-tension and diamond specimens. (1) In rotary bend testing, wire samples were prestrained in tension from 0 to 10 with $2 \%$ increments before subjecting to the fully reversed loading ( $0 \%$ mean strain) at different strain amplitudes. The fatigue life improves with amount of prestrain and increases substantially when the material starts deforming plastically. (2) In the more challenging test setup of tension-tension fatigue, the wire specimens were prestrained in tension $(4,6,9,10$ and $11 \%$ ) and released to $2 \%$ mean strain. Samples were tested at different strain amplitudes to obtain the S-N curve for individual prestrains. Though the resulting strain amplitudes were smaller compared to RBF test, the effect of prestrain on improvement in fatigue life was more significant than the RBF test. The $9 \%$ prestrain fatigue limit increased by $100 \%$ compared to the $4 \%$ prestrain fatigue limit. (3) In diamond specimen testing representative of laser-cut medical devices, the specimens were prestrained in both tension and compression to $9 \%$ before subjecting them to duty cycle at $3.5 \%$ mean strain. If prestraining leads to compressive residual stresses at the locations that will be exposed to tension during cycling, the fatigue life improves, and if prestraining leads to tensile residual stresses at the locations that will be exposed to tension during cycling, it accelerate fracture. Finite-element models demonstrate that large residual stresses are created surrounding an inclusion or void when prestrained past the plasticity region of the material.

Prestraining can lead to improvement or be detrimental depending on the sequence in which the specimens are prestrained and cycled. The superelastic properties of Nitinol create unusually large residual stresses after inhomogeneous deformations compared to traditional metals. Inhomogeneities in deformation can arise from obvious macroscopic inhomogeneities such as bending or torsion, but can also occur from voids, hard inclusions, and even the crystallographic alignment of grains. Of particular note are that stress concentration sites such as inclusions and voids which can be made less potent or more potent depending upon the deformation history of the material. For example, the act of crimping a stent into a catheter creates residual stresses that are protective in a compressive pulsatile fatigue environment, but may lose their protective nature, or even aggravate damage, in other fatigue modes. This highlights the importance of assuring that fatigue tests are preceded by appropriate deformations, and it highlights the risks or extrapolating fatigue data from one test mode to other modes-for example, axial fatigue data to a crushing, or pulsatile duty cycle. While this complicates medical device design and testing, it also provides designers with a very powerful tool to optimize fatigue performance.

\section{Acknowledgment}

The authors acknowledge the support of Ich Ong and Lot Vien in performing the experimental testing and sample preparation for this study.

\section{Open Access}

This article is distributed under the terms of the Creative Commons Attribution 4.0 International License (http://creativecommons.org/ licenses/by/4.0/), which permits unrestricted use, distribution, and reproduction in any medium, provided you give appropriate credit to the original author(s) and the source, provide a link to the Creative Commons license, and indicate if changes were made.

\section{References}

1. A.R. Pelton, V. Schroeder, M.R. Mitchell, X.-Y. Gong, M. Barney, and S.W. Robertson, Fatigue and Durability of Nitinol Stents, J. Mech. Behav. Biomed. Mater., 2008, 1(2), p 153-164

2. A.R. Pelton, Nitinol Fatigue: A Review of Microstructures and Mechanisms, J. Mater. Eng. Perform., 2010, 20(4-5), p 613-617

3. A.L. McKelvey and R.O. Ritchie, Fatigue-Crack Growth Behavior in the Superelastic and Shape-Memory Alloy Nitinol, Metall. Mater. Trans. A, 2001, 32(13), p 731-743

4. R.O. Ritchie, Mechanisms of Fatigue Crack Propagation in Metals, Ceramics and Composites: Role of Crack Tip Shielding, Mater. Sci. Eng. A, 1988, 103(1), p 15-28

5. A. Shamimi, T. Duerig, B. Amin-Ahmadi, and A. Stebner, The Effect of Low Temperature Aging and the Evolution of R-Phase in Ni-Rich NiTi, Shap. Mem. Superelasticity, 2018, 4(4), p 417-427

6. M.E. Launey, I.Ong, and A.R. Pelton, On The Life Fatigue of Superelastic Nitinol, in International Conference on Shape Memory and Superelastic Technologies, 2013, Prague, Czech Republic

7. H. Sehitoglu and S. Alkan, Recent Progress on Modeling Slip Deformation in Shape Memory Alloys, Shap. Mem. Superelast., 2018, 4(1), p 11-25

8. H.M. Paranjape, A.P. Stebner, and K. Bhattacharya, A Macroscopic Strain-Space Model of Anisotropic, Cyclic Plasticity with Hardening, Int. J. Mech. Sci., 2018, 149, p 365-372

9. J.E. Schaffer, Mechanical Conditioning of Superelastic Nitinol Wire for Improved Fatigue Resistance, J. ASTM Int., 2010, 7(5), p 1-7

10. B.A. Dooley, C.C. Lasley, M.R. Mitchell, R.R. Steele and E.M. Tittelbaugh, Shape Memory Alloy Articles with Improved Fatigue Performance and Methods Therefor, United States Patent Application Number, US20040216814A1, 2003

11. A.R. Pelton, B. Clausen, and A.P. Stebner, In-Situ Neutron Diffraction Studies of Increasing Tension Strains of Superelastic Nitinol, Shape Mem. Superelast., 2015, 1(3), p 375-386

12. Y. Zhang, Z. Moumni, J. Zhu, and W. Zhang, Effect of the Amplitude of the Training Stresses on the Fatigue Lifetime of NiTi Shape Memory Alloys, Scripta Mater, 2018, 149, p 66-69

13. S. Gupta, A.R. Pelton, J.D. Weaver, X.Y. Gong, and S. Nagaraja, High Compressive Prestrains Reduce the Bending Fatigue Life of Nitinol Wire, J. Mech. Behav. Biomed. Mater, 2015, 44, p 96-108

14. Standard Test Method for Transformation Temperature of NickelTitanium Alloys by Thermal Analysis, ASTM F 2004-16. (ASTM International, 2000)

15. T.W. Duerig, A.R. Pelton, and K. Bhattacharya, The Measurement and Interpretation of Transformation Temperatures in Nitinol, Shape Mem. Superelast., 2017, 4, p 485-498

16. A.R. Pelton, J. Fino-Decker, L. Vien, C. Bonsignore, P. Saffari, M. Launey, and M.M.R. Mitchell, Rotary-Bending Fatigue Characteristics of Medical-Grade Nitinol Wire, J. Mech. Behav. Biomed. Mater., 2013, 27, p 19-32

17. B. Reedlun, C.B. Churchill, E.E. Nelson, J.A. Shaw, and S.H. Daly, Tension, Compression, and Bending of Superelastic Shape Memory Alloy Tubes, J. Mech. Phys. Solids, 2014, 63, p 506-537 
18. R. Marrey, B. Baillargeon, M.L. Dreher, J.D. Weaver, S. Nagaraja, N. Rebelo, and X.Y. Gong, Validating Fatigue Safety Factor Calculation Methods for Cardiovascular Stents, ASME. J Biomech Eng, 2018, 140(6), p 9

19. S.W. Robertson, M. Launey, O. Shelley, I. Ong, L. Vien, K. Senthilnathan, P. Saffari, S. Schlegel, and A.R. Pelton, A Statistical Approach to Understand the role of Inclusions on the Fatigue Resistance of Superelastic Nitinol Wire and Tubing, J. Mech. Behav. Biomed. Mater, 2015, 51, p 119-131

20. A. Coda, M. Urbano, A. Cadelli, F. Sczerzenie, and S. Beretta, The Effect of Inclusions on Fatigue Behavior of Nitinol and NiTi Shape Memory Alloys for Biomedical Applications, Technical Presentation, International Conference on Shape Memory and Superelastic Technologies, 2013, Prague, Czech Republic

21. A. Toro, F. Zhou, M.H. Wu, W.V. Geertrudyen, and W.Z. Misiolek, Characterization of Non-Metallic Inclusions in Superelastic NiTi Tubes, J. Mater. Eng. Perform., 2009, 18(5-6), p 448-458

22. M. Rahim, J. Frenzel, M. Frotscher, J. Pfetzing-Micklich, R. Steegmüller, M. Wohlschlögel, H. Mughrabi, and G. Eggeler, Impurity Levels and Fatigue Lives of Pseudoelastic NiTi Shape Memory Alloys, Acta Mater., 2013, 61(10), p 3667-3686

23. J.E. Schaffer and D.L. Plumley, Fatigue Performance of Nitinol Round Wire with Varying Cold Work Reductions, J. Mater. Eng. Perform., 2009, 18, p 563-568

24. J.A. Moore, D. Frankel, R. Prasannavenkatesan, A.G. Domel, G.B. Olson, and W. Kam, A Crystal Plasticity-Based Study of the Relationship Between Microstructure and Ultra-high-Cycle Fatigue Life in Nickel Titanium Alloys, Int. J. Fatigue, 2016, 91, p 183-194
25. K. Senthilnathan, P. Saffari, S.W. Robertson, A.R. Pelton, and J. Pfetzing, Influence of Inclusions on localized Stress/Strain Distributions, Technical Presentation, International Conference on Shape Memory and Superelastic Technologies, 2014, Pacific Grove, USA

26. H.M. Paranjape, P.P. Paul, H. Sharma, P. Kenesei, J.-S. Park, T.W. Duerig, L.C. Brinson, and A.P. Stebner, Influences of granular constraints and surface effects on the heterogeneity of elastic, superelastic, and plastic responses of polycrystalline shape memory alloys, J Mech. Phys. Solids, 2017, 102, p 46-66

27. P. Prevey, N. Jayaraman, and R. Ravindranath, Introduction of Residual Stresses to Enhance Fatigue Performance in the Initial Design, in ASME. Turbo Expo: Power for Land, Sea, and Air, vol. 2 (2004), pp. 231-239

28. S. Kalluri, G.R. Halford, and M.A. McGaw, Prestraining and Its Influence on Subsequent Fatigue Life, in NASA Technical Memorandum 106881 (1995)

29. J.C. Le Roux, S. Taheri, J.P. Sermage, J. Colin, and A. Fatemi, Cyclic Deformation and Fatigue Behaviors of Stainless Steel 304L Including Mean Stress and PreStraining Effects, ASME. Pressure Vessels and Piping Conference, 2008, 3, p 411-420

30. C. Bonsignore, Present and Future Approaches to Lifetime Prediction of Superelastic Nitinol, Theoret. Appl. Fract. Mech., 2017, 92, p 298305

Publisher's Note Springer Nature remains neutral with regard to jurisdictional claims in published maps and institutional affiliations. 